\title{
Fetal and placental weight relationships in the rat at Days 13 and 17 of gestation
}

\author{
Neroli A. Norman and N. W. Bruce \\ Department of Anatomy and Human Biology, University of Western Australia, Nedlands, \\ Western Australia 6009
}

\begin{abstract}
Summary. Mean fetal and placental weights were, respectively, 0.018 and $0.051 \mathrm{~g}$ on Day 13 and 0.376 and $0.250 \mathrm{~g}$ on Day 17. Fetal and placental weights within litters were weakly correlated on Day $13(r=0.322)$ but not on Day 17. Litter size was negatively correlated with placental weight on Day $17(r=-0.485)$ but not with fetal weight. Male fetuses were heavier than female fetuses on Day 17 but their placentas were not significantly different. Fetuses and placentas were lighter at the ovarian end of the uterine horn on both days examined, revealing an early influence of local environmental factors on their growth.
\end{abstract}

\section{Introduction}

A number of factors, including litter size, fetal position in the uterine horn (Barr, Jensh \& Brent, 1970), fetal sex (Bruce \& Norman, 1975), and perhaps placental weight (Norman \& Bruce, 1979), affect fetal weight in the rat by term. However, the influence of these factors earlier in gestation has received little attention. In the present study we examined fetal and placental weights at Day 13, the earliest practicable stage, and Day 17 of gestation, and compared the results with those previously found at Day 22 (Norman \& Bruce, 1979).

\section{Materials and Methods}

Nulliparous albino Wistar rats, 3-5 months old and weighing $214 \pm 2.7 \mathrm{~g}$ (mean \pm s.e.m.), were caged with males overnight and vaginal smears were taken each morning. The rats came from a closed breeding colony of 20 years' standing. The day on which spermatozoa were found was called Day 1 of gestation; rats from this colony normally litter on the morning of Day 23. Rats were killed with sodium pentobarbitone between $09: 20$ and 18:00 h on Days 13 or 17 at intervals of approximately 90 and $45 \mathrm{~min}$, respectively. The uterus was opened and conceptuses (fetuses, fetal membranes and placentas) were removed in order, beginning at the ovarian end of the left horn and ending at the ovarian end of the right horn. Each conceptus was opened, examined and weighed before the next conceptus was removed. Only live fetuses and their placentas were weighed; recently dead fetuses were opaque rather than clear, and no heart-beat could be elicited. Any implantation site with a metrial gland but not a live fetus was classed as a 'dead fetus' in the 'Results'.

A $25 \mathrm{~mm}^{2}$ section of amnion was removed for sex determination and the fetus and placenta were then carefully dissected free from the placental membranes and umbilical cord. Day-17 fetuses and Day-13 and -17 placentas were uniformly wiped on a plastic surface to remove excess moisture. The Day-13 fetuses were too fragile to be wiped and so were gently lowered 7 times onto a glass surface to remove excess moisture. Fetuses and placentas were then immediately weighed to the nearest $\mathrm{mg}$ to avoid further moisture loss. The section of amnion was 
examined for sex chromatin, using the staining technique described by Moore \& Barr (1955). The fetus was classified as female if at least 20 Barr bodies were found after a brief scan of the amnion. Fetal sex could not be determined in 12 out of 220 and 10 out of 255 fetuses examined at Days 13 and 17, respectively. The technique was verified by the correct classification of 24 Day- 22 fetuses already sexed by external genitalia.

Analyses of co-variance and common correlation coefficients (Snedecor \& Cochran, 1967) were used to assess fetal weight, and placental weight relationships within litters and within uterine horns, thereby eliminating possible confounding effects due to differences in means between rats and between uterine horns within rats, respectively. The importance of such considerations has previously been pointed out by McLaren (1965). To determine whether overcrowding in the horn affected fetal or placental weights, the difference between the number of live fetuses in the left and right horns of each rat was correlated with the difference between their mean fetal or placental weights. The effect of fetal position in the horn was determined by twoway analyses of variance and, when these were significant, by least significant difference tests carried out on fetal and placental weights at the ovarian end, the middle position and the vaginal end of the horn. When significant, the difference in weight between any two positions was expressed as a percentage of the mean weight in the horn.

\section{Results}

Results are summarized in Table 1 and included for comparison are data on weights at Day 22 of gestation derived from earlier work (Norman \& Bruce, 1979). Over the 9-day period (Days 13-22) fetal weight increased 255 -fold, placental weight 9-fold, and the ratio of fetal weight to placental weight 28 -fold. There was a significant, albeit weak, correlation between fetal and placental weight within litters and within horns within litters at Day 13 and, as previously reported, at Day 22, but not at Day 17.

The time of maternal death, 09:20 to 18:00 h, was related to mean fetal weight in the litter at Day $13(r=0.682, P<0.01)$ and Day $17(r=0.452, P<0.05)$ so mean fetal weights used for remaining correlation analyses were corrected by linear regression equations to the mean time of maternal death $(12: 30 \mathrm{~h})$.

The number of fetuses in the litter (litter size) was negatively correlated with placental weight at Days 17 and 22 of gestation but there was no relationship between litter size and fetal weight. The uterine horn with the greater number of conceptuses did not have significantly different fetal or placental weights at any stage of gestation. Male fetuses were heavier than female fetuses at Days 17 and 22 but their placental weights were similar. Fetal weight was least at the ovarian end of the uterine horn at all 3 stages of gestation, and a similar trend was evident in placental weight. There were no differences in weights at the middle and vaginal end positions of the horn.

\section{Discussion}

Of major interest was the finding that the fetus and placenta at the ovarian end of the uterine horn were lighter than those at the middle or vaginal positions at all stages examined but was most pronounced at Day 13. In the rabbit, the fetus at the ovarian end of the horn is heaviest, but this effect is barely evident until near term and is preceded and perhaps caused by increased placental weight and maternal placental blood flow (Bruce \& Abdul-Karim, 1973). A different explanation may hold for the rat since not only was fetal weight affected much earlier in gestation but placental weight was affected to a lesser extent. We are currently investigating the possible role of variation in the initial time of implantation at different sites along the horn. 
Table 1. Fetal and placental weight and their relationships on Days 13 and 17 of gestation in the rat

\begin{tabular}{|c|c|c|c|}
\hline & Day 13 & Day 17 & Day $22 \dagger$ \\
\hline No. of rats & 23 & 25 & 34 \\
\hline Live fetuses/litter & $12.4 \pm 0.4$ & $10.6 \pm 0.4$ & $9.9 \pm 0.6$ \\
\hline Dead fetuses/litter & $1.2 \pm 0.3$ & $0.7 \pm 0.2$ & $0.9 \pm 0.2$ \\
\hline Male fetuses (\% of total) & $49 \cdot 0$ & $50 \cdot 2$ & 49.7 \\
\hline Mean fetal wt (g) & $0.018 \pm 0.001$ & $0.376 \pm 0.006$ & $4.598 \pm 0.056$ \\
\hline Mean placental wt (g) & $0.051 \pm 0.005$ & $0.250 \pm 0.004$ & $0.463 \pm 0.006$ \\
\hline Fetal wt $(\mathrm{g})$ : placental wt $(\mathrm{g})$ ratio & $0.353 \pm 0.017$ & $1.538 \pm 0.023$ & $10.05 \pm 0.22$ \\
\hline \multicolumn{4}{|l|}{ Correlation of fetal and placental wt: } \\
\hline Within rat & $0.322(251)^{* *}$ & $0.043(239)$ & $0.297(302)^{* *}$ \\
\hline Within uterine horns & $0.305(230)^{* *}$ & $0.022(215)$ & $0.342(270)^{* *}$ \\
\hline \multicolumn{4}{|c|}{ Correlation of no. of live fetuses/litter and } \\
\hline Mean fetal wt & 0.263 & 0.115 & 0.074 \\
\hline Mean placental wt & 0.049 & $-0.485^{*}$ & $-0.528^{* *}$ \\
\hline \multicolumn{4}{|c|}{$\begin{array}{l}\text { Mean male-mean female wt expressed as a \% of } \\
\text { the mean wt within each uterine horn: }\end{array}$} \\
\hline No. of uterine horns & 33 & 45 & 55 \\
\hline Fetal wt & $-1.7 \pm 2 \cdot 6$ & $2.8 \pm 1.1^{*}$ & $5.4 \pm 0.7^{* *}$ \\
\hline Placental wt & $-1.2+1.8$ & $-2.2+1.9$ & $1.3+1.7$ \\
\hline \multicolumn{4}{|c|}{$\begin{array}{l}\text { Weight differences between positions in the } \\
\text { uterine horn expressed as \% of the mean } \\
\text { wt within each horn }\end{array}$} \\
\hline No. of uterine horns & 42 & 49 & 57 \\
\hline \multicolumn{4}{|l|}{ Middle-ovarian position } \\
\hline Fetal wt & $22 \cdot 0 \pm 3 \cdot 4^{* * *}$ & $4 \cdot 4 \pm 2 \cdot 0^{*}$ & $6.4+1 \cdot 2^{* * *}$ \\
\hline Placental wt & $5 \cdot 4+2.5^{*}$ & $6 \cdot 6+2.7^{*}$ & $2 \cdot 8+2.2$ \\
\hline \multicolumn{4}{|l|}{ Vaginal-ovarian position } \\
\hline Fetal wt & $20 \cdot 1 \pm 3 \cdot 6^{* * *}$ & $5 \cdot 1 \pm 1 \cdot 7^{* *}$ & $4 \cdot 3 \pm 1 \cdot 4^{* *}$ \\
\hline Placental wt & $6 \cdot 3+2 \cdot 8^{*}$ & $4 \cdot 2+2.9$ & $4.8+1.9^{*}$ \\
\hline
\end{tabular}

Values given are mean \pm s.e.m. except where otherwise indicated. Values in parentheses refer to the no. of observations.

Significance levels refer to correlation coefficients or to differences between males and females within uterine horns (paired $t$ tests) or to differences between position in the uterine horn (two-way analyses of variance and least significant difference tests): ${ }^{*} P<0.05 ;{ }^{* *} P<0.01 ;{ }^{* * *} P<0.001$.

$\dagger$ Values derived from the work of Norman \& Bruce (1979).

Litter size is negatively related to fetal weight near term in many species (see Dawes, 1968) but the position in the rat is less clear with Barr et al. (1970) and Bruce (1976) reporting a negative relationship, and Watts (1935) and Campbell, Innes \& Kosterlitz (1953) reporting no relationship. A greater number of fetuses might be expected to affect fetal weight at a systemic level, for example by increasing competition for the total nutrient pool, or at a local level by increasing demand on the limited blood supply to each uterine horn. In the present study, there was no evidence for either a systemic or a local effect on fetal weight. On the other hand, litter size was negatively related to placental weight at both Day 17 and Day 22 . This may relate to the endocrine function of the placenta. Individual placental mass tends to increase as litter size decreases in the rat, perhaps to maintain a sufficient total mass of tissue to meet a presumed requirement for placental hormones (Csapo \& Csapo, 1973).

Male fetuses were heavier than female fetuses as early as Day 17 when both were still less than $9 \%$ of their weight near term. There was no apparent difference at Day 13: however, gonadal differentiation occurs around this time (Picon, 1976) and any possible influence of fetal sex steroids on growth would have had little effect. Placental weight was not comparably increased in the Day-17 male fetuses, even though the placenta is largely composed of fetal tissue. The more rapid growth of male fetuses does not seem to depend upon improved placental function, as judged from placental weight, nor is there any evidence that the greater antigenic 
dissimilarity of the male fetus to its mother induces a larger placental mass (Ounsted \& Ounsted, 1970).

The positive relationship between fetal and placental weight near term is much weaker in the rat than in other comparable species (Norman \& Bruce, 1979). One hypothesis advanced for such relationships is that rapid fetal growth near term is limited by placental function as reflected by weight. It might be expected, then, that relationships earlier in gestation when the fetus is associated with a much greater placental mass would be less evident. But in the rat the degree of association of fetal and placental weight estimated from $r^{2}$ was similar at Day $13(10 \%)$ and Day $22(9 \%)$, yet insignificant at Day 17. The following is a possible explanation for this trend. Early in gestation, fetal position in the horn and perhaps differences in the time of initial implantation influence fetal and placental weights in the same direction. This, together with the fact that the fetus and placenta have tissues of common genetic origin may have led to the weak relationship found at Day 13. By Day 17 these factors have less influence or some degree of catch-up growth has occurred, as seems to be so with the position in the horn effect. Other factors, such as fetal sex, increase the variance in fetal weight without influencing placental weight and so further obscure any relationship. Later in gestation the original hypothesis, that rapid fetal growth is limited by placental function, begins to apply, leading to the weak relationship observed at Day 22.

Whatever the explanation, the divergence in the strength of fetal weight-placental weight relationships at different stages of gestation, and between different species (Norman \& Bruce, 1979) suggests that many factors have a role in its origin. From the present results it is now clear that a relationship may exist early in gestation and, furthermore, that at least one environmental factor, position in the uterine horn, begins to influence fetal weight before the latter has reached $0.4 \%$ of its value near term.

We are very grateful to Mr E. Hunt and Mr S. Parkinson for technical assistance.

\section{References}

Barr, M., Jr, Jensh, R.P. \& Brent, R.L. (1970) Prenatal growth of the albino rat: effects of number, intrauterine position and and resorptions. $A \mathrm{~m} . \mathrm{J}$. Anat. 128, 413-428.

Bruce, N.W. (1976) The distribution of blood flow to the reproductive organs of rats near term. $J$. Reprod. Fert. 46, 359-362.

Bruce, N.W. \& Abdul-Karim, R.W. (1973) Relationships between fetal weight, placental weight and maternal placental circulation in the rabbit at different stages of gestation. J. Reprod. Fert. 32, 1524.

Bruce, N.W. \& Norman, N. (1975) Influence of sexual dimorphism on foetal and placental weights in the rat. Nature, Lond. 257, 62-63.

Campbell, R.M., Innes, I.R. \& Kosterlitz, H.W. (1953) Some dietary and hormonal effects on maternal, foetal and placental weights in the rat. J. Endocr. 9, 68-75.

Csapo, A.I. \& Csapo, E.F. (1973) Ovariectomy induced placental hypertrophy. Prostaglandins 4, 189-200.

Dawes, G.S. (1968) Foetal and Neonatal Physiology. $A$ Comparative Study of the Changes at Birth, Ch. 4, pp. 42-59. Yearbook Medical Publishers, Chicago.
McLaren, A. (1965) Genetic and environmental effects on foetal and placental growth in mice. $J$. Reprod. Fert. 9, 79-98.

Moore, K.L. \& Barr, M.L. (1955) Smears from the oral mucosa in the detection of chromosomal sex. Lancet ii, 57-58.

Norman, N.A. \& Bruce, N.W. (1979) Fetal and placental weight relationships in the albino rat near term. Teratology 19, 245-250.

Ounsted, C. \& Ounsted, M. (1970) Effect of Y chromosome on fetal growth-rate. Lancet ii, 857858.

Picon, R. (1976) Testosterone secretion by foetal rat testes in vitro. $J$. Endocr. 71, 231-238.

Snedecor, G.W. \& Cochran, W.G. (1967) Statistical Methods, 6th edn, pp. 381-446. Iowa State University Press, Ames.

Watts, R.M. (1935) The effect of administration of preparations of growth hormone of the anterior lobe of the pituitary upon gestation and the weight of the newborn (Albino rats). Am. J. Obstet. Gynec. 30, 174-185.

Received 16 January 1979 